\title{
Communication
}

\section{Safety of Oral Food Challenges in Early Life}

\author{
Katherine Anagnostou 1,2 \\ 1 Section of Immunology, Allergy and Rheumatology, Department of Pediatrics, Texas Children's Hospital, \\ Houston, TX 77030, USA; Aikaterini.Anagnostou@bcm.edu; Tel.: +1-832-824-1319 \\ 2 Section of Immunology, Allergy and Rheumatology, Department of Pediatrics, Baylor College of Medicine, \\ Houston, TX 77030, USA
}

Received: 10 May 2018; Accepted: 28 May 2018; Published: 30 May 2018

check for updates

\begin{abstract}
Oral food challenges are becoming more frequent in the allergy clinic due to an increased demand related to early food introduction in infants. We examined the safety of oral food challenges in 18 high-risk infants with prior allergic reactions, as well as infants with no known exposure to the food, presenting consecutively in a dedicated food allergy clinic for an oral food challenge. Foods challenged included peanut, tree nuts, sesame, baked egg, baked milk, and soy. A total of 17/18 $(94 \%)$ infants had a negative challenge. Only $1 / 18(6 \%)$ had a positive challenge, and in this case, symptoms were mild and limited to the skin. Our results suggest that food challenges in infants and young children up to the age of 2 years are safe with symptoms limited to the skin when reactions occur. In our cohort, the large majority of food challenges were negative, with most infants being sensitized rather than allergic to the food. Larger studies are needed to confirm this finding.
\end{abstract}

Keywords: oral food challenge; food allergy; infant; anaphylaxis

\section{Introduction}

Food allergies are common and increasing in prevalence, representing a major health concern in many countries around the world. In the United States, approximately $4-8 \%$ of children are affected, with the prevalence of food allergy being highest in infants and toddlers, with the ' 8 major allergenic foods' in childhood being milk, egg, peanut, tree nuts, shellfish, fish, wheat, and soy [1]. Food allergies pose a significant burden on the affected patients and their families [2]. Dietary restrictions, high levels of anxiety, and social limitations are common problems resulting in decreased quality of life. It is therefore of crucial importance to make the correct diagnosis and, currently, the gold standard test for food allergy diagnosis is an 'oral provocation challenge' or 'food challenge'. An oral food challenge (OFC) is the process of administering gradually increasing doses of a food allergen up to a full age-appropriate portion, under medical supervision. Patients are observed for any signs or symptoms of an allergic reaction, which would be interpreted as a 'positive' challenge ('allergic patient'). If no signs or symptoms occur during dose administration and for $2 \mathrm{~h}$ after ingesting a full portion of the food, the challenge is considered 'negative' and the patient not allergic [3].

Oral food challenges in infants and young children are becoming an increasingly common occurrence in the allergy clinic. This is the result of various factors including (a) recent significant changes in our approach to allergenic food introduction with the aim to prevent the development of food allergy; (b) reported important advantages in baked milk and egg introduction into the diet of milk and egg allergic children; and (c) importance of differentiating between sensitization (positive skin tests or specific IgE to the food without clinical reactivity) and true allergy (clinical reactivity to the food) in early life. 


\section{Methods}

Our study examined the safety of infant food challenges. The study was approved by the Institutional Research Board committee at Baylor College of Medicine (protocol H-40400). We have established an in-hospital dedicated pediatric clinic, available since May 2017, to encourage early allergenic food introduction in high-risk infants that would be able to tolerate this. Infants are defined as 'high-risk' if they suffer from moderate/severe or early onset eczema, an existing food allergy, or both. The clinic also supports introduction of baked milk and egg to infants with the aim to liberate the diet and accelerate tolerance. Food challenges are also performed in order to differentiate between sensitization and true allergy in very young age.

We collected data prospectively on the first 18 patients, up to the age of 2 years, who underwent food challenges, with the objective to evaluate safety of this procedure in this age group. All food challenges were performed in accordance with the PRACTALL guidelines [3]. Materials used for food challenges included smooth peanut or tree nut butter (diluted in milk, juice, or smoothie), tree nut milk (i.e., almond milk), cupcake containing milk or egg (for the baked challenges), soy milk, and tahini (for sesame). Skin prick testing to both the food extract and the fresh food were performed on the day of the challenge. For baked challenges, skin prick testing also included the baked form of the food (milk or egg). All necessary safety measures were in place prior to conducting the challenges. A dedicated clinic area was used, within the existing allergy clinic for challenges. Resuscitation facilities were available in case of severe allergic reactions, as well as access to a fully staffed Pediatric Intensive Care Unit (PICU) in case of severe anaphylaxis. (on a different floor of the same hospital where the clinic is located). Both physician and nursing cover was available for the challenges. (ratio: 1 physician and 1 nurse per 3-4 patients).

\section{Results}

We prospectively collected data on the first 18 children, up to the age of 2 years, who underwent an open, graded, oral food challenge (OFC) in our clinic for this study (see Table 1).

In this cohort, median age was 13 months, range: 8 months-2 years. Male to female ratio was 8:1.

With regards to atopic history, the overwhelming majority of our patients $(16 / 18,89 \%)$ had eczema, but only 2 had a history of wheeze and only 1 a history of allergic rhinitis. A total of 14/18 (78\%) reported another food allergy (different from the challenge food). Diagnosis of all atopic conditions were made and documented previously by an allergist.

Foods challenges included peanut in 6 children (33\%), baked milk in $3(17 \%)$, soy in $2(11 \%)$, almond in $2(11 \%)$, baked egg in $1(5 \%)$, hazelnut in $1(5 \%)$, walnut in $1(5 \%)$, cashew in $1(5 \%)$, and sesame in $1(5 \%)$.

Median skin prick test (SPT) values and ranges were: $3 \mathrm{~mm}$ for the food extract (range 0-20 mm), $0 \mathrm{~mm}$ for the fresh food (range 0-18 mm), and $7 \mathrm{~mm}$ for the baked form of the food tested (range 0-9 mm). Specific IgE values were available for 16/18 infants prior to the challenge. Median value was $0.34 \mathrm{kU} / \mathrm{L}$ (range $0.1-11.1 \mathrm{kU} / \mathrm{L}$ ).

A total of $17 / 18(94 \%)$ infants had a negative challenge. Only $1 / 18(6 \%)$ had a positive challenge and in this case, symptoms were mild and limited to the skin (erythema and hives). Symptoms occurred within 1 hour and were treated successfully with a single dose of oral antihistamine (Benadryl). There were no biphasic reactions.

In 11/18 (61\%), the food had not been ingested previously. Patients with a prior history of allergic reaction reported the following symptoms: urticaria (5/18), vomiting (1/18), eczema flare (2/18), difficulty breathing (2/18), angioedema (1/18), itching (1/18), irritability $(1 / 18)$. The patient who had a positive challenge reported hives only with his initial reaction. Both infants with reported difficulty in breathing as part of their initial reaction had negative challenges to the food. 
Table 1. Infant oral food challenges: demographics, atopic history and oral food challenge information.

\begin{tabular}{|c|c|c|c|c|c|c|c|c|c|c|c|c|}
\hline $\mathbf{N}$ & Age (Months) & Gender & $r$ Food & SPT Extract (mm) & SPT Fresh $(\mathrm{mm})$ & Specific IgE (kU/L) & Eczema & Allergic Rhinitis & Asthma & Other Food Allergy & Symptoms of Index Reaction & Challenge Result \\
\hline 1 & 13 & M & $\begin{array}{c}\text { Baked } \\
\text { milk }\end{array}$ & 6 & 10 & 1.63 & No & No & No & Egg & Hives & Negative \\
\hline 2 & 19 & M & Baked & 8 & 10 & 0.63 & Yes & No & No & No & Hives, angioedema, vomiting & Negative \\
\hline 3 & 8 & M & Peanut & 3 & - & 0.1 & Yes & No & No & No & No prior ingestion & Negative \\
\hline 4 & 14 & M & $\begin{array}{l}\text { Baked } \\
\text { milk }\end{array}$ & 10 & 18 & 0.2 & Yes & No & No & Egg, peanut & Eczema flare & Negative \\
\hline 5 & 15 & M & Soy & 20 & 1 & 0.26 & Yes & No & No & Egg, peanut & No prior ingestion & Negative \\
\hline 6 & 22 & M & Baked egg & 0 & 0 & 11.1 & No & Yes & No & Milk & Hives & Positive \\
\hline 7 & 13 & M & Soy & 0 & 20 & 0.89 & Yes & No & No & Egg, peanut, wheat & Hives, difficulty in breathing & Negative \\
\hline 8 & 10 & M & Peanut & 0 & 0 & 0.12 & Yes & No & No & Egg & No prior ingestion & Negative \\
\hline 9 & 14 & M & Sesame & 0 & 3 & 0.94 & Yes & No & No & Egg, peanut, wheat & Hives, difficulty in breathing & Negative \\
\hline 10 & 10 & M & Peanut & 3 & 0 & & Yes & No & No & Milk & $\begin{array}{l}\text { No prior ingestion } \\
\text { Non }\end{array}$ & Negative \\
\hline 11 & 10 & $\mathrm{~F}$ & Almond & 2 & 0 & 0.21 & Yes & No & No & Peanut & No prior ingestion & Negative \\
\hline 12 & 12 & M & Almond & 3 & 0 & 0.34 & Yes & No & No & Egg, peanut, wheat & No prior ingestion & Negative \\
\hline 13 & 15 & M & Peanut & 4 & 4 & & Yes & No & No & No & No prior ingestion & Negative \\
\hline 14 & 12 & M & Peanut & 2 & 0 & 0.55 & Yes & No & No & Tree nuts & No prior ingestion & Negative \\
\hline 15 & 34 & M & Hazelnut & 2 & 0 & 1.34 & Yes & No & No & Walnut, pecan & No prior ingestion & Negative \\
\hline 16 & 25 & M & Walnut & 3 & 0 & 0.1 & Yes & No & Yes & Egg, peanut & No prior ingestion & Negative \\
\hline 17 & 27 & M & Cashew & 5 & 4 & 0.37 & Yes & No & Yes & Egg, peanut & No prior ingestion & Negative \\
\hline 18 & 8 & $\mathrm{~F}$ & Peanut & 1 & 0 & 0.1 & Yes & No & No & No & Eczema flare, itching, irritability & Negative \\
\hline
\end{tabular}

M: Male, F: Female. 


\section{Discussion}

There have been significant changes recently in the recommendations for infant weaning, with early allergenic food introduction strongly encouraged, especially with regards to peanut, contrary to previous recommendations. A consensus document [4] published in January 2017 advises peanut introduction into the infant diet between the ages of 4 and 11 months. This recommendation is particularly relevant for high-risk infants, defined as those with severe eczema, egg allergy, or both. This recent change in practice, also supported by the American Academy of Pediatrics, was based on the results of the Learning Early About Peanut (LEAP) clinical trial [5] which showed that early introduction of peanut in high-risk infants resulted in significantly reduced risk of peanut allergy development by age 5 years.

Following publication of the new guidelines for peanut introduction, we anticipated an increasing number of peanut challenges in infants and young children, especially those belonging to the 'high-risk' category, and set up an early introduction clinic to meet this demand. Peanut was the most common food challenged in our clinic cohort. All our patients had negative peanut challenges. Most (83\%) had never ingested peanut previously, but all had moderate-severe eczema and 3 had another food allergy (egg, milk, and tree nut). Skin tests to peanut extract revealed results of $0-4 \mathrm{~mm}$, but interestingly, we noted mostly (67\%) negative skin tests to the fresh form of peanut (usually peanut butter). The same observation was made for most of the tree nuts challenged (hazelnut, almond, and walnut). This may reflect a better predictive value for the fresh form of nut (compared to the extract), but larger patient groups will be required to further investigate this finding.

In addition to peanut, we opted to include baked milk and egg challenges, as research suggests that up to $70 \%$ of milk- and egg-allergic children are able to tolerate the baked form of these foods [6]. This allows for a more liberated diet, nutritional benefits, less social restrictions, and, ultimately, better quality of life. Regular ingestion of baked milk or egg has also been associated with a faster development of tolerance to the fresh form of the food [7]. However, no universally accepted cut-offs for either skin prick test or specific IgE that may predict which children are able to tolerate the baked form of milk or egg exist, so a baked milk or egg challenge remains the gold standard diagnostic tool for baked milk or egg tolerance. Four of our patients were challenged to baked milk (3/18) and baked egg $(1 / 18)$. The baked egg challenge resulted in the only positive challenge in our cohort. It involved a 22-month old male with prior history of hives on exposure to cooked egg. He reacted after consuming $145 \mathrm{mg}$ of egg protein, with hives on his anterior chest and facial erythema. Following treatment with a single dose of antihistamine all his symptoms resolved.

Infant food challenges differ from those that involve older children or adults. In fact, a AAAAI (American Academy of Allergy, Asthma and Immunology) work group report was produced with the aim to guide clinicians in the conduction of OFCs to peanut in infants [8]. The main concern of parents and clinicians is the possibility of anaphylaxis during an infant OFC, especially because in this age group, the diagnosis of anaphylaxis may present some added diagnostic challenges. Very young children are unable to describe certain symptoms such as pruritus or throat tightness. Certain signs such as irritability and behavioral changes can be difficult to interpret as they may also occur in healthy infants [9]. In young children aged 0-2 years, cow's milk and egg are the most commonly reported foods implicated in anaphylactic episodes [10], followed by peanuts, sesame, and fish [9,11]. We did not observe any episodes of anaphylaxis in our cohort of patients and the only positive challenge resulted in mild cutaneous symptoms.

\section{Conclusions}

We suggest that food challenges in infants and young children up to the age of 2 years are generally safe; symptoms appear to be limited to the skin when reactions occur. This includes high-risk infants with prior allergic reactions as well as infants with no known exposure to the food challenged. In carefully selected populations, the large majority of food challenges are likely to be negative, 
with most infants being sensitized rather than allergic to the food. However, our patient sample is small and further studies will be required to confirm these findings.

Author Contributions: Anagnostou performed all food challenges in the study, wrote and reviewed the manuscript.

Conflicts of Interest: The author has no conflicts of interest to disclose.

\section{References}

1. Jones, S.M.; Burks, A.W. Food Allergy. N. Engl. J. Med. 2017, 377, 1168-1176. [CrossRef] [PubMed]

2. Sicherer, S.H.; Noone, S.A.; Munoz-Furlong, A. The impact of childhood food allergy on quality of life. Ann. Allergy Asthma Immunol. 2001, 87, 461-464. [CrossRef]

3. Sampson, H.A.; Van Wijk, R.G.; Bindslev-Jensen, C.; Sicherer, S.; Teuber, S.S.; Burks, A.W.; Dubois, A.E.J.; Beyer, K.; Eigenmann, P.A.; Werfel, T.; et al. Standardizing double-blind, placebo-controlled oral food challenges: American Academy of Allergy, Asthma \& Immunology-European Academy of Allergy and Clinical Immunology PRACTALL consensus report. J. Allergy Clin. Immunol. 2012, 130, 1260-1274. [PubMed]

4. Togias, A.; Cooper, S.F.; Acebal, M.L.; Assa'ad, A.; Baker, J.R., Jr.; Beck, L.A.; Block, J.; Byrd-Bredbenner, C.; Chan, E.S.; Eichenfield, L.F.; et al. National Institute of Allergy \& Infectious Diseases (NIAID) Addendum Guidelines for the Prevention of Peanut Allergy in the United States: Report of the National Institute of Allergy and Infectious Diseases-sponsored expert panel. J. Allergy Clin. Immunol. 2017, 139, 29-44. [PubMed]

5. Du Toit, G.; Roberts, G.; Sayre, P.H.; Bahnson, H.T.; Radulovic, S.; Santos, A.F.; Brough, H.A.; Phippard, D.; Basting, M.; Turcanu, V.; et al. Randomized Trial of Peanut Consumption in Infants at Risk for Peanut Allergy. N. Engl. J. Med. 2015, 372, 803-813. [CrossRef] [PubMed]

6. Lemon-Mulé, H.; Sampson, H.A.; Sicherer, S.H.; Shreffler, W.G.; Noone, S.; Nowak-Wegrzyn, A. Immunologic changes in children with egg allergy ingesting extensively heated egg. J. Allergy Clin. Immunol. 2008, 122, 977-983. [CrossRef] [PubMed]

7. Leonard, S.A.; Caubet, J.C.; Kim, J.S.; Groetch, M.; Nowak-Wegrzyn, A. Baked milk- and egg-containing diet in the management of milk and egg allergy. J. Allergy Clin. Immunol. Pract. 2015, 3, 13-23. [CrossRef] [PubMed]

8. Bird, J.A.; Groetch, M.; Allen, K.J.; Bock, S.A.; Leonard, S.; Nowak-Wegrzyn, A.H.; Sicherer, S.; Clark, A.; Fleischer, D.M.; Vickery, B.; et al. Conducting an Oral Food Challenge to Peanut in an Infant. J. Allergy Clin. Immunol. Pract. 2016, 5, 1-12. [CrossRef] [PubMed]

9. Simons, F.E.R.; Sampson, H.A. Anaphylaxis: Unique aspects of clinical diagnosis and management in infants (birth to age 2 years). J. Allergy Clin. Immunol. 2015, 135, 1125-1131. [CrossRef] [PubMed]

10. Alonso, T.; Moro Moro, M.; García, M.; Esteban Hernandez, J.; Rosado Ingelmo, A.; Vila Albelda, C.; Gomez Traseira, G.; Cardenas Contreras, R.; Sanz Sacristán, J.; Hernandez Merino, A.; et al. Incidence of anaphylaxis in the city of Alcorcon (Spain): A population-based study. Clin. Exp. Allergy 2012, 42, 578-589. [CrossRef]

11. Allen, K.J.; Koplin, J.J. The Epidemiology of IgE-Mediated Food Allergy and Anaphylaxis. Immunol. Allergy Clin. N. Am. 2012, 32, 35-50. [CrossRef] [PubMed]

(C) 2018 by the author. Licensee MDPI, Basel, Switzerland. This article is an open access article distributed under the terms and conditions of the Creative Commons Attribution (CC BY) license (http://creativecommons.org/licenses/by/4.0/). 